\title{
Pharmacokinetics of chewed vs. swallowed raltegravir in a patient with AIDS and MAI infection: some new conflicting data
}

Christoph D Spinner ${ }^{1,2^{*}}$, Florian Wille ${ }^{3}$, Christiane Schwerdtfeger ${ }^{1,2}$, Philipp Thies ${ }^{1}$, Ursula Tanase ${ }^{1}$, Guido Von Figura ${ }^{1}$, Roland M Schmid ${ }^{1}$, Werner J Heinz ${ }^{4}$ and Hartwig Hf Klinker ${ }^{4^{*}}$

\begin{abstract}
Background: While HIV, AIDS and atypical Mycobacterium infections are closely linked, the use of Integrase-Inhibitor based cART, notably raltegravir-based regimens is more widespread. RAL should be double-dosed to $800 \mathrm{mg}$ semi-daily in situation of rifampicin co-medication, because RAL is more rapidly metabolized due to rifampicin-induced Uridine-5'-diphosph- gluronosyl-transferase (UGT1A1). Recently, it was speculated that chewed RAL might lead to increased absorption, which might compensate the inductive effect of rifampicin-rapid metabolized RAL, as part of cost-saving effects in countries with high-tuberculosis prevalence and less economic power.
\end{abstract}

Methods: We report measurement of raltegravir pharmacokinetics in a 34-year AIDS-patient suffering from disseminated Mycobacterium avium infection with necessity of parenteral rifampicin treatment. RAL levels were measured with HPLC (internal standard: carbamazepine, LLQ $11 \mathrm{ng} / \mathrm{ml}$, validation with Valistat 2.0 program (Arvecon, Germany)). For statistical analysis, a two-sided Wilcoxon signed rank test for paired samples was used.

Results: High intra-personal variability in raltegravir serum levels was seen. Comparable $C_{\text {max }}$ concentrations were found for $800 \mathrm{mg}$ chewed and swallowed RAL, as well as for $400 \mathrm{mg}$ chewed and swallowed RAL. While $\mathrm{C}_{\max }$ seems to be more dependent from overall RAL dosing than from swallowed or chewed tablets, increased $A U C_{12}$ is clearly linked to higher RAL dosages per administration. Anyway, chewed raltegravir showed a rapid decrease in serum levels.

Conclusions: We found no evidence that chewed $400 \mathrm{mg}$ semi-daily raltegravir in rifampicin co-medication leads to optimized pharmacokinetics. There is need for more data from randomized trials for further recommendations.

Keywords: HIV, Chewed, Raltegravir, Mycobacterium avium, Pharmacokinetic

\section{Background}

While Human Immunodeficiency Virus (HIV), Aquired Immunodeficiency Syndrome (AIDS) and atypical Mycobacteriosis infections are closely linked, the use of Integrase-Inhibitor (INI) based antiretroviral regiments (ART), notably raltegravir (RAL)-based regiments is more widespread during HAART era. Raltegravir's benefit is the favorable drug-interaction profile whilst treating AIDS-defining

\footnotetext{
* Correspondence: christoph.spinner@tum.de; klinker_H@ukw.de 'Department of Medicine II, University Hospital Klinikum rechts der Isar, Ismaningerstr 22, 81675 Munich, Germany

${ }^{4}$ Department of Medicine II, Division of Infectious Diseases, University Of Wuerzburg Medical Center, Oberduerrbacherstr 6, 97080 Wuerzburg, Germany

Full list of author information is available at the end of the article
}

events in HIV- patients [1]. Rifampicin is known to be active against atypical Mycobacterial infections, including Mycobacterium avium infections [2]. [3] Nevertheless, RAL is more rapidly metabolized in case of rifampicin co-administration due to rifampicin-induced Uridine-5'-diphosphgluronosyl-transferase (UGT1A1) [1,4]. Therefore, it is recommended to double-dose RAL to $800 \mathrm{mg}$ semi-daily (BID) in case of Rifampicin-use, which causes high additional costs in these patients [4]. Recently, there has been conflicting data, which suggests using a standard-dose of $400 \mathrm{mg}$ RAL BID, taken chewed as part of an ART, might, in the event of Rifampicin co-administration, lead to higher drug absorption and lower drug intersubject pharmacokinetic variability $[5,6]$. As reported recently, it was 
speculated that chewed RAL might lead to increased absorption, which might thus compensate the inductive effect of rapid metabolized RAL due to rifampicin and therefore have cost-saving effects in countries with high-tuberculosis prevalence and less economic power [5]. Despite that aspect, some data for high inter- and intra-personal variability in pharmacokinetics were reported for HIV-patients in general [7]. Lack of sufficient RAL levels were not clearly associated with virological failure of antiretroviral therapy, but that there might be some influences of overall plasma exposure of the drug $[7,8]$. Therefore, conflicting data in differential RAL dosages play a major role in decisions on clinical HIV-patient care and overall antiretroviral therapy success.

\section{Clinical objectives}

We report our experience with a recent admission of a 34-year-old Caucasian male HIV-patient, who was diagnosed as being HIV/HBV-co-infected 5 years prior to actual presentation. CDC Stage was C3, WHO stage IV due to thrush esophagitis and systemic cytomegalovirus reactivation. $\mathrm{CD} 4$-nadir $=8$ cells $/ \mu \mathrm{l}$, he was known to belong to the risk group of men having sex with men.

4 months before current presentation at our center, the patient was diagnosed with mycobacterium avium infection in a lymph node biopsy whilst having fever, weight loss and general lymphadenopathy in a peripheral hospital. Antiretroviral therapy with co-formulated Tenofovir disoproxil fumarate and Emtricitabine (TVD) in combination with integrase-inhibitor RAL was initiated. Oral antimicrobial chemotherapy with rifabutin, ethambutol and clarithromycin was started without delay. The patient was discharged from the peripheral hospital. Subsequently, persistent fever and weight loss appeared within the first months.

The clinical situation worsened despite medical therapy. He was admitted to our emergency department with shock and severe hypoglycemia 4 months after initiation of the first antimycobacterial treatment. The marasmic patient (body mass index of 15), suffered from 5 liters of watery diarrhea per day, severe hypoalbuminemia (albumin 1,4 $\mathrm{g} / \mathrm{dl}$ ), protein deficiency (serum protein $4,4 \mathrm{~g} / \mathrm{dl}$ ), and iron deficiency anemia (hemoglobin 7,0 g/dl). Subsequently, mycobacterium avium was diagnosed by microscopy and culture in samples of pleural effusion, stool, sigmoid biopsy and peripheral blood. No other microorganisms could be identified in blood culture diagnostics. Due to the lack of intestinal absorption, antimicrobial chemotherapy was escalated to parenteral rifampicin (600 mg once daily), ethambutol (400 mg three times daily), clarithromycin (500 mg semi-daily) and amikacin (750 mg once daily).

Before adjusting ART to the recommended dose of $800 \mathrm{mg}$ RAL BID (adult formulation) in combination with Tenfovir disoproxil fumarate co-formulated with
Table 1 Pharmacokinetic data of Raltegravir after administration of different dosages of raltegravir by chewing or swallowing the drug in a patient with AIDS and MAI infection

\begin{tabular}{|c|c|c|c|c|}
\hline \multirow[t]{2}{*}{ Parameter } & \multicolumn{4}{|c|}{ Raltegravir concentration (ng/ml) } \\
\hline & $\begin{array}{l}400 \mathrm{mg} \\
\text { swallowed }\end{array}$ & $\begin{array}{l}400 \mathrm{mg} \\
\text { chewed }\end{array}$ & $\begin{array}{l}800 \mathrm{mg} \\
\text { swallowed }\end{array}$ & $\begin{array}{l}800 \mathrm{mg} \\
\text { chewed }\end{array}$ \\
\hline $\mathrm{C}_{\mathrm{Oh}}$ & 66 & $<45$ & 439 & 47 \\
\hline $\mathrm{C}_{1 \mathrm{~h}}$ & 884 & 865 & 511 & 676 \\
\hline $\mathrm{C}_{2 \mathrm{~h}}$ & 904 & 930 & 781 & 660 \\
\hline $\mathrm{C}_{4 h}$ & 699 & 207 & 1055 & 1147 \\
\hline $\mathrm{C}_{8 \mathrm{~h}}$ & 71 & 173 & 1032 & 723 \\
\hline $\mathrm{C}_{12 \mathrm{~h}}$ & 62 & 94 & 885 & 372 \\
\hline$A \cup C_{0-4}$ & 2972 & 2489 & 2957 & 2837 \\
\hline $\mathrm{AUC}_{12}$ & 4778 & 3783 & 10965 & 8767 \\
\hline $\begin{array}{l}\text { Difference } \text { AUC }_{12} \\
(\mathrm{ng} / \mathrm{ml}) ; \text { median; min; max }\end{array}$ & \multicolumn{2}{|l|}{$-3,5 ;-102 ; 492$} & \multicolumn{2}{|l|}{$215 ;-165 ; 513$} \\
\hline (p-value) & \multicolumn{2}{|l|}{ (p-value 0,84$)$} & \multicolumn{2}{|l|}{$(p$-value 0,22$)$} \\
\hline
\end{tabular}

Emtricitabine, subsequent pharmacokinetic studies were performed after informed consent of the patient. In doing so, RAL concentrations were measured in serum before, 1, 2, 4, 8 and 12 hours after a BID oral application of RAL with a dosage of $400 \mathrm{mg}$ and $800 \mathrm{mg}$. Before new measurement after every dose adjustment, a lead-in phase of more than 24 hours was performed. For optimized reliability, chewed RAL dosage studies were performed semidaily at different points of time. At any time, a standard meal was provided and pantoprazole was co-administered at a dosage of $40 \mathrm{mg}$ once daily. Usage of $\mathrm{pH}$-altering agents like pantoprazole was associated with a possible increase in AUC and $C_{\max }$ in recent studies [9]. However, the clinical impact of $\mathrm{pH}$-altering agents and RAL levels

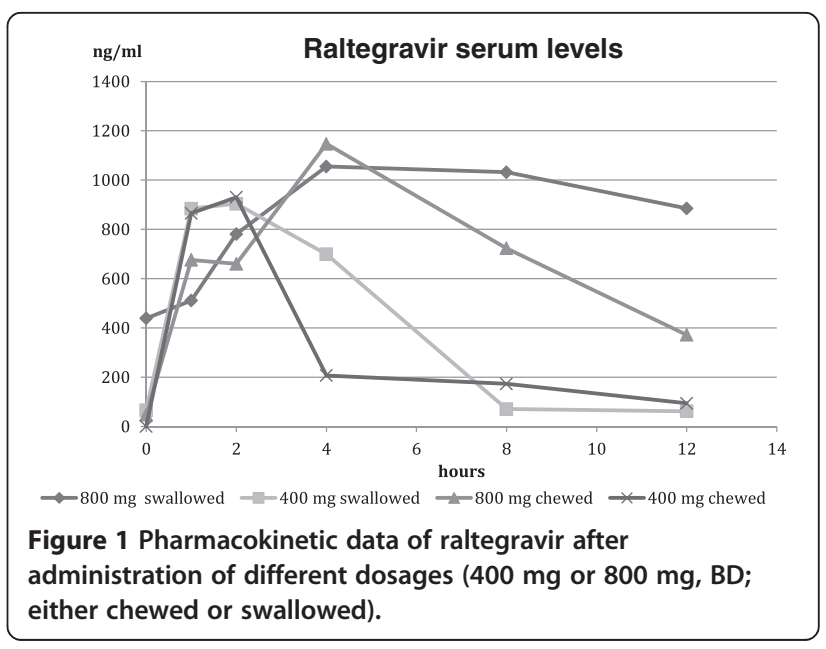


was less important in HIV/AIDS patients, compared to healthy volunteers [10].

\section{Methods}

RAL levels were measured with high performance liquid chromatography (HPLC, internal standard: carbamazepine, LLQ $11 \mathrm{ng} / \mathrm{ml}$, validation with Valistat 2.0 program (Arvecon, Germany)). For statistical analysis, a two-sided Wilcoxon signed rank test for paired samples was used.

\section{Results}

Overall, comparable RAL $\mathrm{C}_{2 \mathrm{~h}}$ concentrations were found with either chewed or swallowed $400 \mathrm{mg}$ or $800 \mathrm{mg}$ of RAL BID in the case of rifampicin co-administration. Similarly $\mathrm{C}_{\max }$ concentrations were found for $800 \mathrm{mg}$ chewed and swallowed RAL BID, as well as for $400 \mathrm{mg}$ chewed and swallowed RAL BID. While $\mathrm{C}_{\max }$ seems to be more dependent on overall RAL dosing than on swallowed or chewed tablets, increased $\mathrm{AUC}_{12}$ is clearly linked to higher RAL dosages per administration. Chewed or swallowed RAL in a dosage of $800 \mathrm{mg}$ BID led to acceptable $\mathrm{AUC}_{12}$ levels (8767 ng/ml; $10965 \mathrm{ng} / \mathrm{ml})$, whereas $400 \mathrm{mg}$ RAL BID showed significantly lower $\mathrm{AUC}_{12}$ levels when chewed or swallowed (3783 ng/ml; $4778 \mathrm{ng} / \mathrm{ml}$ ). Interestingly, lower $\mathrm{AUC}_{12}$ levels were seen in chewed dosages of RAL, compared to a similar dose of swallowed RAL in $400 \mathrm{mg}$ and $800 \mathrm{mg}$. For chewed or swallowed $400 \mathrm{mg}$ RAL dosages, a rapid decrease in serum levels was seen, beginning 2-4 hours after application. These findings were confirmed in low serum levels at the beginning of the next medication at $\mathrm{C}_{0}$ or $\mathrm{C}_{12}$. Our finding results in dangerously low serum concentrations 8-12 hours after application of chewed or swallowed $400 \mathrm{mg}$ RAL BID, as well as in low $\mathrm{C}_{0}$ concentrations $(<45 \mathrm{ng} / \mathrm{ml} ; 66 \mathrm{ng} / \mathrm{ml})$. Notably lower $C_{0} R A L$ levels were also seen for the chewed $800 \mathrm{mg}$ RAL BID dosage when compared to the equivalent chewed $800 \mathrm{mg}$ RAL BID dosage $(47 \mathrm{ng} / \mathrm{ml})$. Although a relevant intra-personal variability in raltegravir serum levels in this HIV-patient with watery diarrhea and intestinal mal-absorption due to systemic Mycobacterium avium infection, have to be taken into account, only swallowed $800 \mathrm{mg}$ RAL led to a lasting RAL serum concentration of $439 \mathrm{ng} / \mathrm{ml}$ at $\mathrm{C}_{0}$ and $885 \mathrm{ng} / \mathrm{ml}$ at $\mathrm{C}_{12}$ with acceptable $\mathrm{AUC}_{12}$ of 10965 $\mathrm{ng} / \mathrm{ml}$. Pharmacokinetic data in detail are shown in Table 1, a graph of RAL pharmacokinetics is shown in Figure 1. However, a comparison of RAL concentrations after chewed and swallowed administration of RAL showed no statistically significance.

\section{Conclusion}

Based on these findings we could not demonstrate optimized RAL levels after taking chewed compared to swallowed RAL tablets in contrast to previously reported data [5]. Even though there might be changes in RAL pharmacokinetics due to pantoprazole co-administration in our data, we would expect a 3-4 fold higher $C_{\max }$ and AUC of RAL levels. It can be speculated that the RAL levels might be less high as here reported in the absence of gastric proton pump inhibitors. This might be another risk factor for lower RAL levels in case of rifampicin coadministration. Anyhow, whilst potential cost-saving effects of RAL dosing savings may be important, the risk of virological failure due to resistance-evolution plays a crucial role in INI-based antiretroviral therapy. Therefore, we keep using double-dosed swallowed RAL tablets in the case of rifampicin co-medicated patients, as recommended by the official prescribing information, until further data from controlled trials are available. Dose reduction can cause relevant and potentially dangerous decreases in RAL serum levels and $\mathrm{AUC}_{12}$, which can cause serious harm to HIV-patients. Potential cost-saving effects should not endanger effective antiretroviral therapy, until there is further evidence of any other superior strategy.

\section{Competing interests}

CDS received grants from AbbVie Germany, Boehringer Ingelheim, Bristol-Meyers Squibb, Gilead Sciences, Janssen-TMC, Pfizer Germany, ViiV Healthcare. CSp is participating in multi-center studies which are supported by AbbVie Germany, Sanofi Aventis, Janssen and ViiV Healthcare. He received Gilead Sciences grants for research work.

HHK received grants, board membership fees and lecture fees from AbbVie, Boehringer, Bristol-Myers Squibb, Gilead, Glaxo-SmithKline, Hexal, Janssen-Cilag, MSD, Roche, and ViV Healthcare.

WJH reieved research and travel grants from MSD/Merck, Pfizer, Alexion, Astellas Pharma, Gilead Sciences, received board membership fees and lecture fees Astellas, Gilead Science, Pfizer and is participating in multi-center studies which are supported by Merck, GSK, Gilead Sciences, Basilea/Astellas, Viropharma.

All other authors are not declaring any conflicts of interests.

\section{Authors' contributions}

All authors were involved in data acquisition and manuscript preparation. CDS and HHK prepared and revised the manuscript in cooperation with all authors. FW performed blood withdrawal and patient management for the study. CS, UT, PT, GVF, RMS and CDS took clinical care for the patient. WJH and HHK performed TDM measurements and statistical calculation. All authors read and approved the final manuscript.

\section{Acknowledgments}

We thank Ms Anne Elter and Ms Diana Schirmer for technical assistance.

\section{Author details}

'Department of Medicine II, University Hospital Klinikum rechts der Isar, Ismaningerstr 22, 81675 Munich, Germany. ${ }^{2}$ Interdisciplinary HIV Centre (IZAR), University Hospital Klinikum rechts der Isar, Ismaningerstr 22, 81675 Munich, Germany. ${ }^{3}$ TUM Medical School, Technische Universitaet Muenchen, Ismaningerstr 22, 81675 Munich, Germany. ${ }^{4}$ Department of Medicine II, Division of Infectious Diseases, University Of Wuerzburg Medical Center, Oberduerrbacherstr 6, 97080 Wuerzburg, Germany.

Received: 18 September 2014 Accepted: 13 December 2014 Published online: 17 January 2015

\section{References}

1. Adams JL, Greener BN, Kashuba ADM: Pharmacology of HIV integrase inhibitors. Curr Opin HIV AIDS 2012, 7:390-400.

2. van Ingen J, Hoefsloot W, Mouton JW, Boeree MJ, van Soolingen D: Synergistic activity of rifampicin and ethambutol against slow-growing 
nontuberculous mycobacteria is currently of questionable clinical significance. Int J Antimicrob Agents 2013, 42:80-82.

3. Zhao $X$, Wang $Y$, Pang Y: Antimicrobial susceptibility and molecular characterization of mycobacterium intracellulare in China.

Infect Genet Evol 2014, 27:332-338.

4. Wenning LA, Hanley WD, Brainard DM, Petry AS, Ghosh $K$, Jin B, Mangin E, Marbury TC, Berg JK, Chodakewitz JA, Stone JA, Gottesdiener KM, Wagner $J$ A, Iwamoto M: Effect of rifampicin, a potent inducer of drugmetabolizing enzymes, on the pharmacokinetics of raltegravir. Antimicrob Agents Chemother 2009, 53:2852-2856.

5. Gervasoni C, Riva A, Impagnatiello C, Galli M, Cattaneo D: Is chewed raltegravir an option to care for HIV-infected patients with active tuberculosis? Clin Infect Dis 2013, 57:480-481.

6. Cattaneo D, Baldelli S, Cerea M, Landonio S, Meraviglia P, Simioni E, Cozzi V, Fucile S, Gazzaniga A, Clementi E, Galli M, Rizzardini G, Gervasoni C: Comparison of the In Vivo Pharmacokinetics and In Vitro Dissolution of Raltegravir in HIV Patients Receiving the Drug by Swallowing or by Chewing. Antimicrobial Agents Chemother 2012, 56(12):6132. doi:10.1128/AAC.00942-12.

7. Cattaneo D, Gervasoni C, Meraviglia P, Landonio S, Fucile S, Cozzi V, Baldelli S, Pellegrini M, Galli M, Clementi E: Inter- and intra-patient variability of raltegravir pharmacokinetics in HIV-1-infected subjects. J Antimicrob Chemother 2012, 67:460-464. doi:10.1093/jac/dkr498.

8. Garrido C, de Mendoza C, Alvarez E, García F, Morello J, Garcia S, Ribera E, Rodríguez-Novoa S, Gutierrez F, Soriano V, Sinres Team: Plasma raltegravir exposure influences the antiviral activity and selection of resistance mutations. AIDS Res Hum Retroviruses 2012, 28:156-164.

9. Iwamoto M, Wenning LA, Nguyen BY, Teppler H, Moreau AR, Rhodes RR, Hanley WD, Jin B, Harvey CM, Breidinger SA, Azrolan N, Farmer HF Jr, Isaacs RD, Chodakewitz JA, Stone JA, Wagner JA: Effects of omeprazole on plasma levels of Raltegravir. Clin Infect Dis 2009, 48:489-492.

10. Brainard DM, Wenning LA, Stone JA, Wagner JA, Iwamoto M: Clinical pharmacology profile of raltegravir, an HIV-1 integrase strand transfer inhibitor. J Clin Pharmacol 2011, 51:1376-1402.

\section{Submit your next manuscript to BioMed Central and take full advantage of:}

- Convenient online submission

- Thorough peer review

- No space constraints or color figure charges

- Immediate publication on acceptance

- Inclusion in PubMed, CAS, Scopus and Google Scholar

- Research which is freely available for redistribution 